\title{
Monitoring and Analyzing of Circadian and Ultradian Locomotor Activity Based on Raspberry-Pi
}

\author{
Vittorio Pasquali ${ }^{1}$, Riccardo Gualtieri ${ }^{2}$, Giuseppe D'Alessandro ${ }^{3}$, Maria Granberg ${ }^{4}$, \\ David Hazlerigg ${ }^{5}$, Marco Cagnetti ${ }^{6}$ and Fabio Leccese ${ }^{6, *}$
}

1 Psychology Department-Neuroscience Section Medicine and Psychology Faculty, "Sapienza" University, Via dei Marsi n.78, 00185 Rome, Italy; vittorio.pasquali@uniroma1.it

2 Department of Physics, University of Illinois at Urbana Champaign, 1110 W Green St., Urbana, 61801 IL, USA; rgualtie@illinois.edu

3 Department of Physics, “Sapienza” University, P.le Aldo Moro 2, 00185 Rome, Italy; giuseppe.dalessandro@uniroma1.it

4 Norwegian Polar Institute, Fram Center, Hjalmar Johansen gt.14, NO-9296 Tromsø, Norway; maria.granberg@npolar.no

5 Department of Arctic and Marine Biology, Faculty of Biosciences, Fisheries and Economy, University of Tromsø, NO-9037 Tromsø, Norway; david.hazlerigg@uit.no

6 Science Department, University of "Roma Tre", Via della Vasca Navale 84, 00146 Rome, Italy; ing.marco.cagnetti@gmail.com

* Correspondence: leccese@uniroma3.it; Tel.: +39-06-57337347

Academic Editors: Simon J. Cox and Steven J. Johnston

Received: 1 June 2016; Accepted: 12 September 2016; Published: 15 September 2016

\begin{abstract}
A new device based on the Raspberry-Pi to monitor the locomotion of Arctic marine invertebrates and to analyze chronobiologic data has been made, tested and deployed. The device uses infrared sensors to monitor and record the locomotor activity of the animals, which is later analyzed. The software package consists of two separate scripts: the first designed to manage the acquisition and the evolution of the experiment, the second designed to generate actograms and perform various analyses to detect periodicity in the data (e.g., Fourier power spectra, chi-squared periodograms, and Lomb-Scargle periodograms). The data acquisition hardware and the software has been previously tested during an Arctic mission with an arctic marine invertebrate.
\end{abstract}

Keywords: Raspberry-Pi; I/O (Input/Output) board; data-logger; locomotor activity; single-board computer

\section{Introduction}

The spontaneous locomotor activity of animals is a useful parameter in ecological studies [1] and in chronobiology, in particular for circadian analysis. Animals show daily rhythms in their various physiological and behavioral functions. These rhythms are synchronized to environmental cycles, such as light-dark cycles (LD), which are related to the Earth's rotation. Rhythmicity may be controlled by a circadian clock located in the central nervous system, such as the optic lobe or central brain mass, depending on the species [2].

The analysis of behavioral rhythmicity has led to the development of specialized hardware and software for a variety of different applications, with target organisms ranging from fruit flies to humans, and data acquisition based on methods including infrared (IR) sensors, accelerometers, and radioand video-tracking [3-8]. Associated with these systems, a range of commercially available analysis packages have been developed to run on Windows or MacOS operating systems [9-11].

In this article, we present a new system for the acquisition and analysis of the behavioral data in chronobiological studies, based on an inexpensive hardware, coupled to a Raspberry-Pi computer 
(Raspberry Pi Foundation, Cambridge, United Kingdom). This configuration provides an inexpensive, platform-independent, and open-source platform to circadian analysis, similar to those utilized in other fields [12-17]. The data acquisition hardware is connected to the Raspberry-Pi computer by an electronic interface designed to operate under field conditions. The software for data acquisition and analysis was developed in the Astrophysics laboratory of Sapienza University in Rome, using a free programming language (Python). The design aim was to integrate algorithms typically used for the analysis of periodic signals with both specific functions for signal filtering, and functions allowing user specification of the statistical stringency in the signal detection. This approach increases flexibility in the analytical capability of the software.

The system has been tested under field conditions in the Svalbard Islands using a marine invertebrate Gammarus Setosus as a model. An image of the field test is also shown.

\section{Monitoring and Data Analysis}

Our system was designed to acquire data on 17 channels, 15 of which are IR activity sensors, and two of which are sensors for environmental light and temperature. As shown in Figure 1, the monitoring system consists of aquaria, electronics mounted on the aquaria and the signal conditioning circuit, light and temperature sensors, and a connector box interfaced with the Raspberry-Pi through a flat cable.

The software is composed of two sections: the first handles the data acquisition and the storage, the second manages the data analysis.

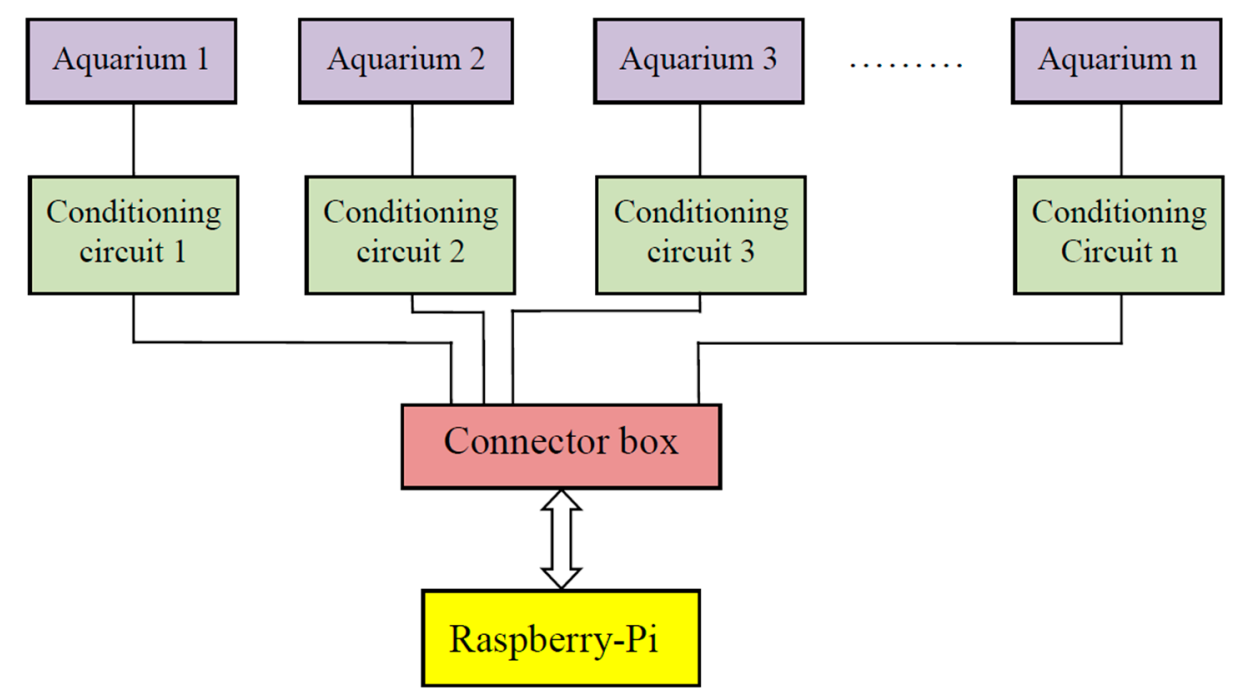

Figure 1. Block diagram of the monitoring system.

\subsection{Aquaria}

This device has been designed for small $(0.5-3 \mathrm{~cm})$ freshwater and sea invertebrates. This device does not filter or oxygenate the water, and therefore relies on regular water changes by the user. The system is built around Corning $225 \mathrm{~cm}^{2}$ culture flasks, which are made of transparent plastic that permits natural or artificial illumination of the aquarium environment. Two squared sheets of transparent Plexiglas (6 mm thick) were cut to match the area of the large faces of the culture flask and glued in place using plastic cement (e.g., Loctite, Super Attack). These sheets were used to locate the IR emitters and sensors on the outside of the aquarium (Figure 2). 


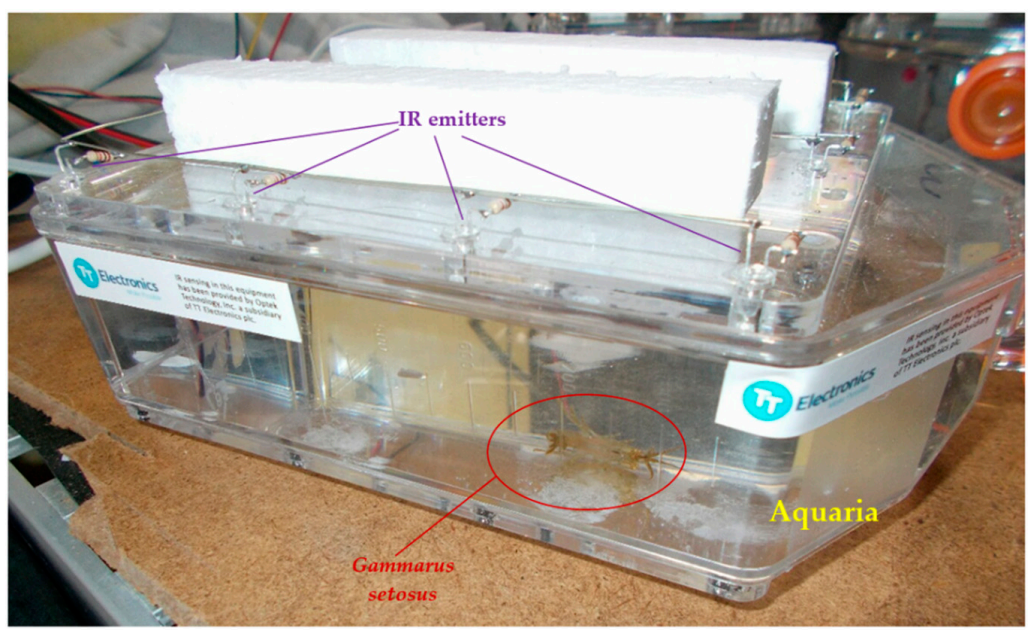

Figure 2. The electronic aquaria, with a Gammarus shrimp inside and with the IR emitters mounted on the aquarium roof. The IR sensors are in the floor of the aquarium (not visible here).

\subsection{Electronics}

The optoelectronics components (OPTEK Inc., Carrollton, TX, USA) were: (a) Ø3 mm GaAlAs plastic IR emitting diodes OP298B narrow irradiance pattern, $\lambda 890 \mathrm{~nm}, \phi 25^{\circ}$, max output power $4.8 \mathrm{~mW} / \mathrm{cm}^{2}$ [18]; (b) $\varnothing 3 \mathrm{~mm}$ NPN plastic silicon phototransistors OP598B wide receiving angle, $\lambda 860 \mathrm{~nm}$, and $\phi 25^{\circ}$ [19] (Figure 2).

These emitter and sensor devices give a narrow beam, necessary for sensitive detection of activity of small invertebrates. Additionally, these devices offer optimal electronic coupling, and have proven high reliability at low temperatures $[18,19]$. The output signal of each sensor was amplified by operational amplifiers MC3303 [20]. This device offers a Gain Bandwidth product and operational temperature range ideal for this application. Then a positive-OR gate (74LS21 [21]) was used to sum all signals. The input signal for the Raspberry-Pi GPIO digital I/O pins was supplied from a LM 555 [22] in mono-stable mode, which provides an impulse of $15 \mathrm{~ms}$ limited to $+3.3 \mathrm{Vdc}$ such that compatibility with the GPIO (General Purpose Input Output) input dynamic is ensured. Figure 3 shows the schematic of the signal conditioning circuit reduced to only two IR sensors.

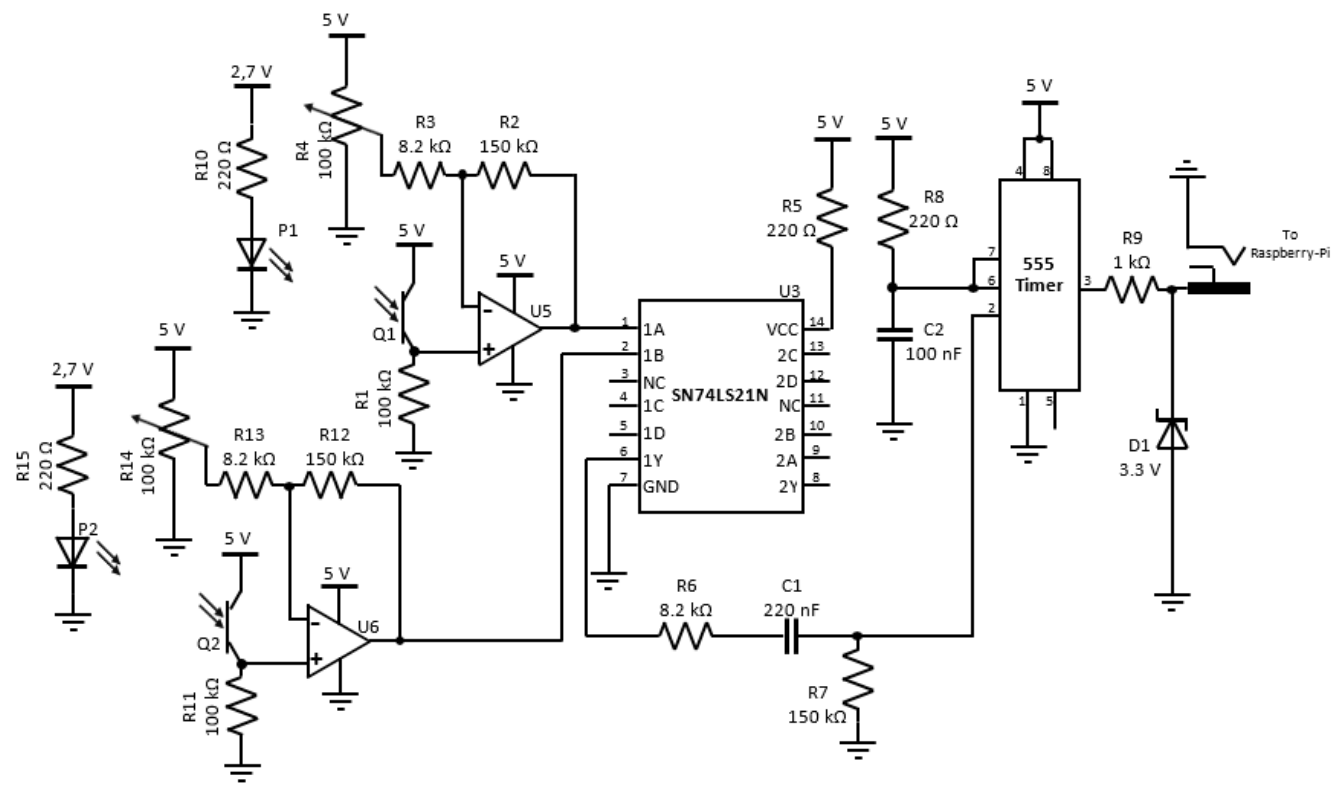

Figure 3. Schematic of the signal conditioning circuit reduced to only two IR sensors. 
Figure 4 shows the conditioning circuit housed in a watertight box.

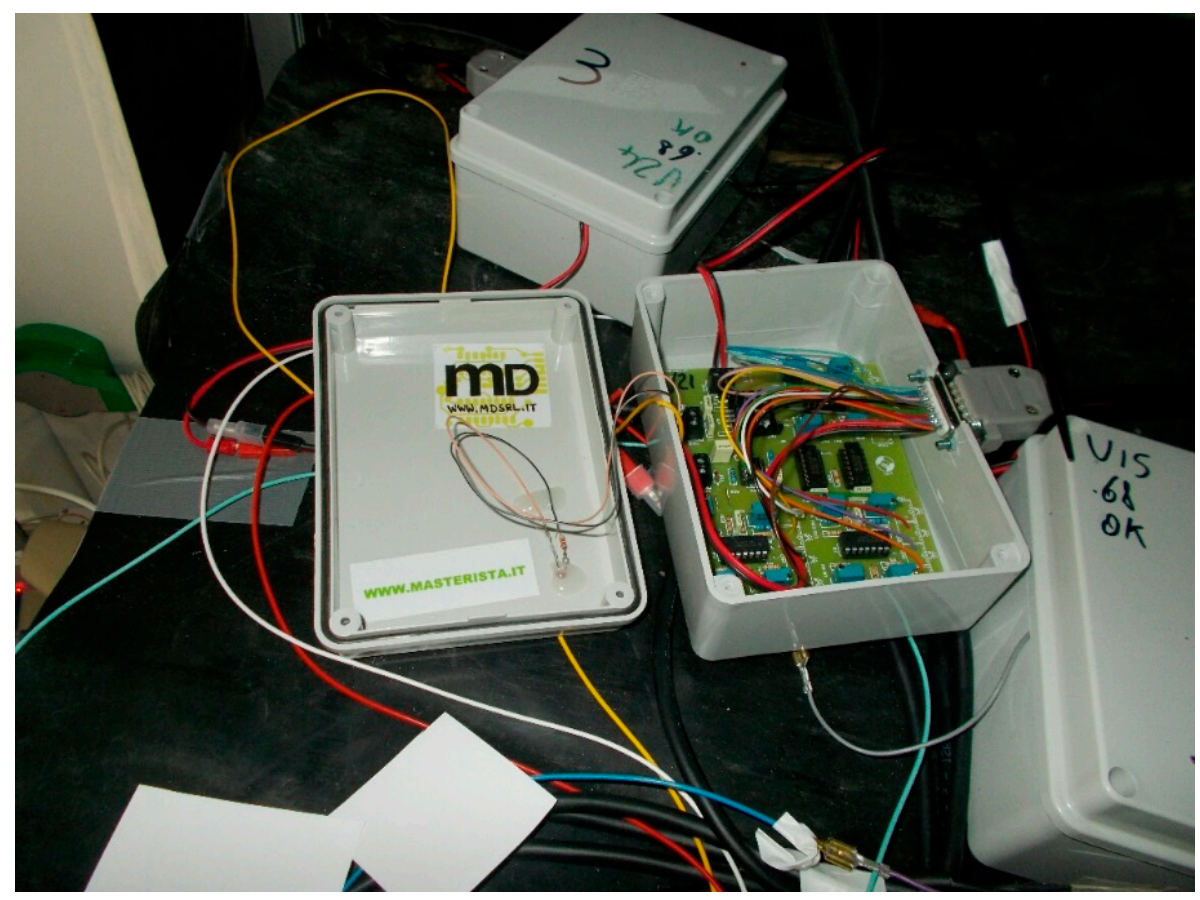

Figure 4. Signal conditioning circuit.

\subsection{Connector Box}

The connector box was built to facilitate the connection between different devices and sensors (e.g., IR devices, radar devices, and light/temperature sensors). It is composed of two lines of PCB (polychlorinated biphenyl) screw connectors and a flat cable connector to pick up the signals and send them to the GPIO of the Raspberry (Figure 5).

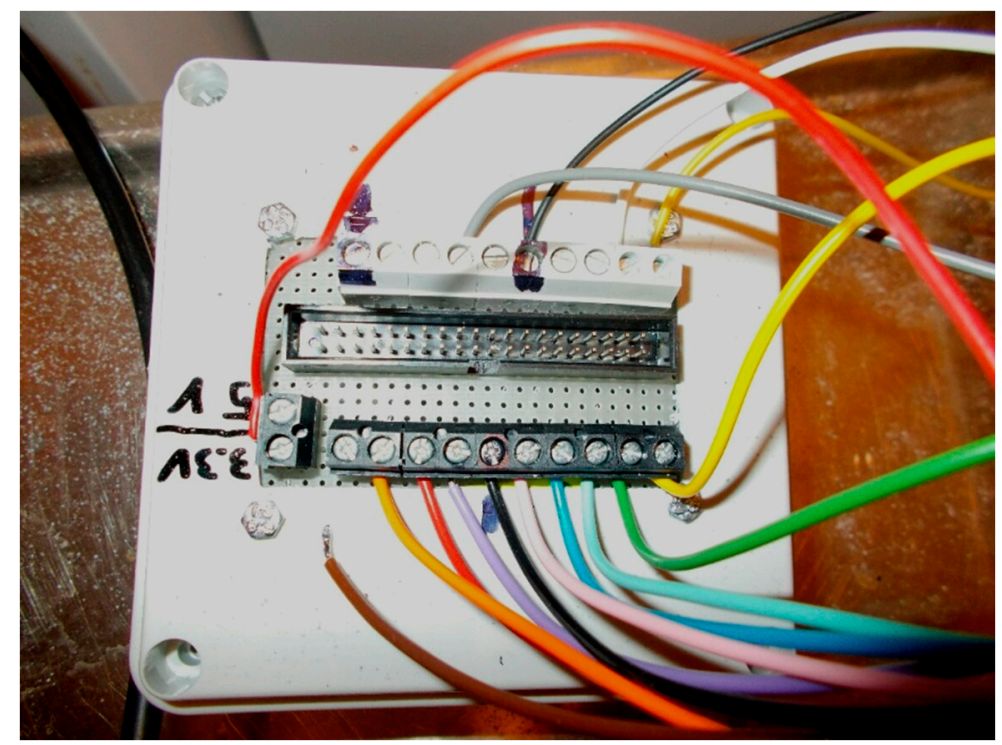

Figure 5. Connector box. Flat cable connector between two line of PCB (polychlorinated biphenyl) screw connectors. 


\subsection{Software Package}

The software package included in our setup consists of two separate scripts designed to interact with each other such that data acquisition and analysis is more easily managed. The acquisition program has the same structure of the one presented in [23] (Pasquali et al., 2016) but is modified to include multiple channels.

The Raspberry Pi has a limited amount of computing resources and the acquisition of several signals has a high computational cost. For this reason, we decided not to include in our package any real-time data visualization tools; nevertheless, the user is able to control the execution of the experiment by examining the main data file that is updated periodically with incoming data.

To achieve this goal, we designed the second script as an on-demand analyzer. The user is able to recall the data for a defined period of acquisition and for selected channels. The software will immediately perform the requested analysis without interrupting the acquisition. This user defined call generates files containing the requested data and plots of the quantities of interest, e.g., data streams, actograms, power spectra and Lomb-Scargle periodogram analysis to catch eventual periods in the data. In this way, the preliminary analysis will be performed on a branch of the main data file. When the on-demand analysis is completed, the user can transfer the output files remotely.

\subsubsection{Main Software}

The main software running on Raspberry is temporized by using a Unix C-Time library [24]. A single internal clock self-generated from another routine, or by an external hardware clock, gives the start for all operations. When the clock rises up, the software reads the sensors and records the binary state of the logic channel to a temporary array. This value is modified every $10 \mathrm{~min}$ and the software accumulates total counts for each channel and writes the results to a dedicated external file. The file is updated and every hour the Raspberry-Pi backs up the data in another file saved on internal SD memory and sends it on Ethernet to remote users. The time series data can be divided into up into four sections for subsequent analysis.

\subsubsection{Real Time Analysis}

The automatic generation of "actograms" can be activated or disabled by the user. The user can specify the length of the time segment and how to bin the dataset for analysis. The subroutine reads the last four chunks of data (one day) and produces four actograms on a table configuration (two rows, two columns) where an actogram is repeated on the second and third plot. To ensure minimal computation time with the hardware limitations, the software performs several controls on the dataset before starting the calculation.

The user can see the plot automatically updated on his screen using a virtual graphics server like Xming [25].

\subsubsection{A Posteriori Analysis}

\section{Total Actogram Generator}

The same subroutine that generates the real time actograms are used to produce the total actogram of the dataset. As example, an output plot is shown in Figure 6 (all underlying data used in this publication can be found here [26]). 


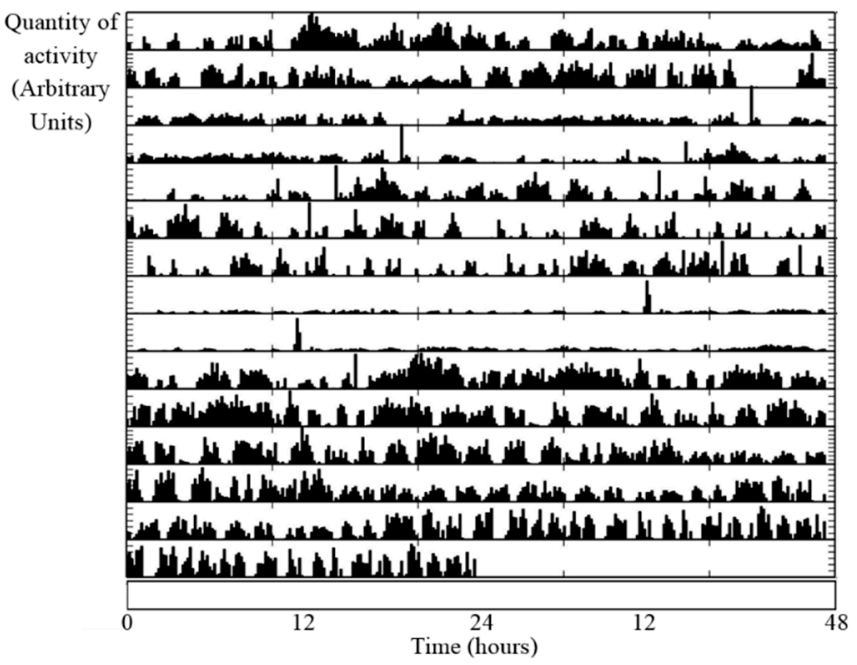

Figure 6. Example of double-plot actograms on the screen of the Raspberry-Pi.

The Fourier Transform Module

One of the steps required in the data analysis of our project is to perform Fourier analysis on the time streams. For this purpose, we developed a Python module that collects the powerful functions distributed under the numpy and scipy packages, which are freely distributed under the GNU (General Public License) license. Once the module is included in the main analysis script, all the functions are ready to be used. Below are descriptions of the functions.

After the main library is fed with the time stream data, an algorithm retrieves the data acquisition rate. This step is fundamental for the construction of the frequency axis, and to evaluate the Discrete Fast Fourier Transform (FFT) of the stream applying a window function. The result is a relative power spectral density (rPSD), and, if not specified, the module uses default values for the spectral range.

Advanced users will be able to choose the window function for their analysis, divide the sample into chunks with lengths corresponding to powers of two, evaluate the FT as the mean value of the chunks, and make an estimation of the response and phase of the complex FT. The outputs of the module are a file, an image for inspection of the results, and a numpy array that can be used for further analysis.

Figure 7 shows an example of the FFT output from this algorithm, where the $x$-axis is time in minutes on a logarithmic scale, and the $y$-axis is the relative magnitude of the peaks, where the total power (i.e., the area under the FFT curve) has been normalized to 100, to calculate relative power spectral densities (rPSD) [27].

\section{Lomb-Scargle Periodogram}

To better study the FT analysis, a statistical test, such as the Lomb-Scargle method [28,29], can be applied.

For this purpose, we have included in our package an easy access to the scipy function for the evaluation of a Lomb-Scargle periodogram. The package calculates the periodogram using a slightly modified algorithm from Townsend [30], which allows the periodogram to be calculated using only a single pass through the input arrays for each frequency. The function takes the time series data array and the sampling frequency and outputs the periodogram formatted as a numpy array. 


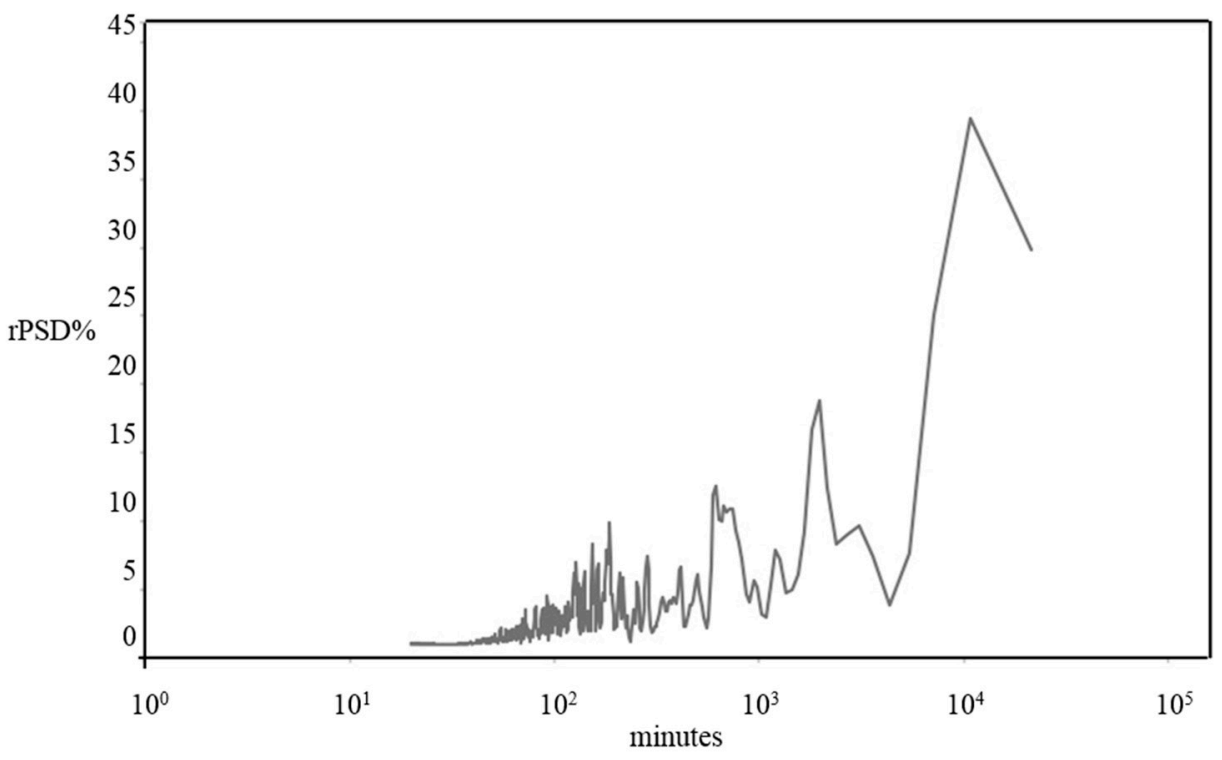

Figure 7. Example of spectral analysis by FT (Fourier Transform). Power values are shown on the $y$-axis; periods (in minute) on a logarithmic scale are shown on the $x$-axis.

\section{Health Monitoring System Subroutines (HMSS)}

The main software has different sub-routines, which are synchronized with the data acquisition but are only executed occasionally. The Raspberry-Pi often works without user control, and thus it must control itself with a subroutine that checks the set-up health state. There are two different categories of HMSS: the first ones are for the external set-up control and the second ones are for the Raspberry-Pi state analysis. The first category of HMSS is:

- Photodiode control: if the logical binary state of photodiode does not change over an extended period, it is probably broken, and a warning message appears on the screen. Using an external GSM (Global System for Mobile Communications) module, the Raspberry-Pi sends an SMS message or an email to the user.

- Battery control: the Raspberry-Pi can be powered by an external battery, so it is important to control the charge state of this battery.

- Physical state of the set-up: the Raspberry-Pi performs a continuous control on intensity of light, temperature, and humidity in the room.

The second category HMSS are:

- Memory control: the Raspberry has a SD memory card on which data is written. If the available memory falls below ten percent, the Raspberry-Pi compresses the data on the SD card. After the compression, the Raspberry-Pi enters a low-resolution mode where it reduces the bit size of data acquired and starts to overwrite the oldest data. A warning message is sent to the user.

- CPU temperature: the Raspberry can operate in strenuous environments, often with a high demand on the CPU, so its temperature is monitored. If the temperature becomes critical (around $90^{\circ} \mathrm{C}$ ), all activities are stopped.

Figure 8 shows the block scheme of the software that runs inside the Raspberry-Pi. 


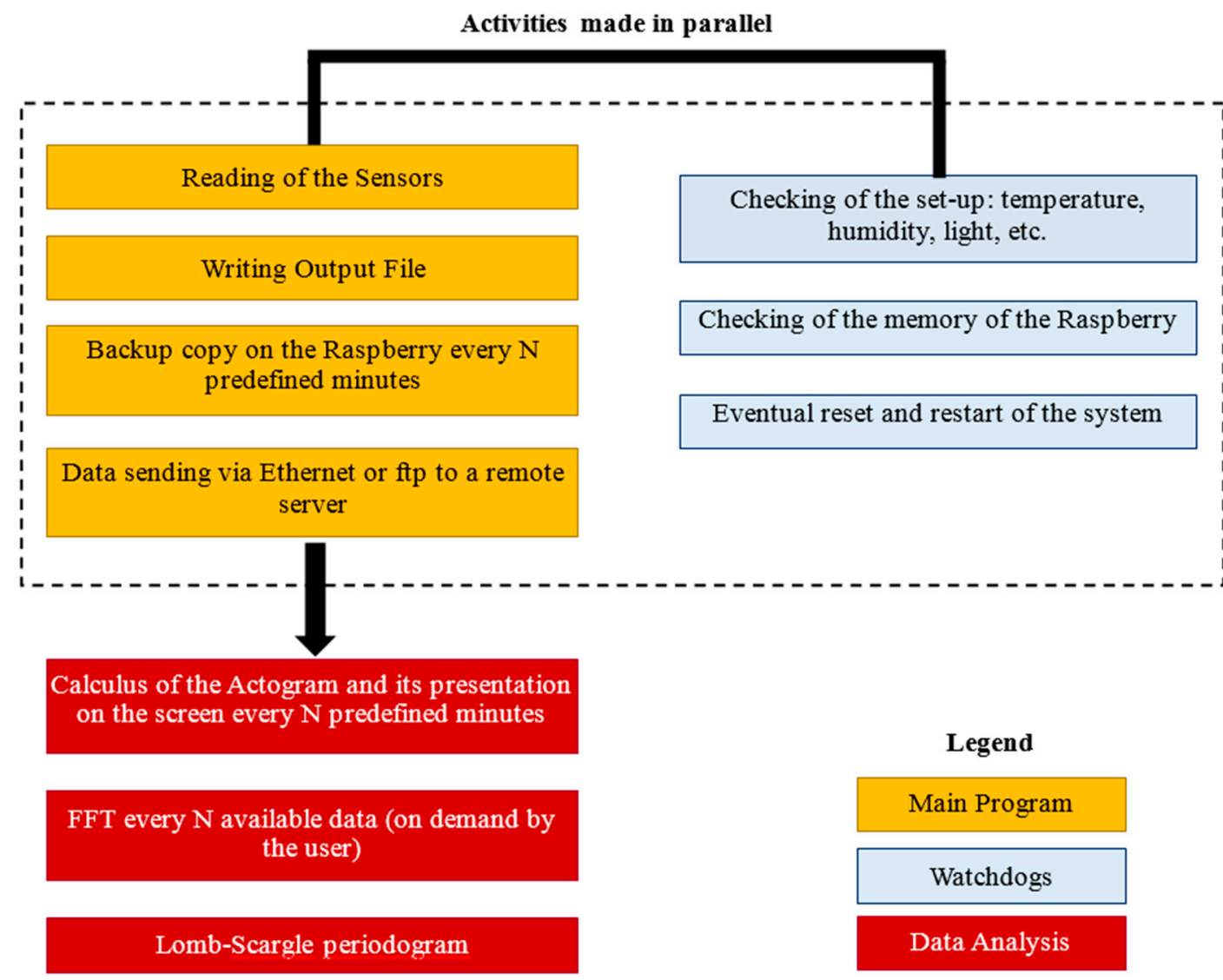

Figure 8. Shows the block scheme of the software that runs inside the Raspberry-Pi.

\section{Comparison with Previous System}

To highlight the advantages offered by this system, a comparison between the actual and the standard system is necessary.

The classical setup of this kind of instrument includes a PC-based measurement chain that drives the hardware section with a custom software. The hardware consists of several aquaria where the animals are located, electronic circuits inside the aquaria for the detection of the animal's motion, and an acquisition card inserted inside of the personal computer that receives the signals coming from the aquaria. The software section manages two different processes: the acquisition and the analysis of the data. The data are processed offline, and this allows researchers more flexibility in configuring both the hardware and software. Figure 9 shows the classical architecture (left side) and the Raspberry-Pi based architecture (right side).
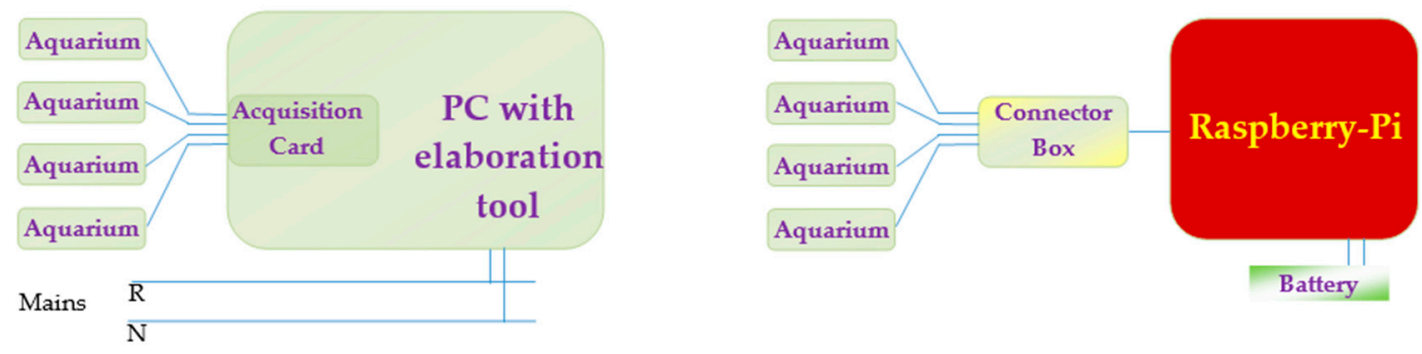

Figure 9. Classical and Raspberry-based architecture.

Although a classical system has the undoubted advantage to be already certified and used by many research groups in the world, it presents several drawbacks: 
(1) The hardware sections, available on the market, are preset and custom configurations are not allowed, e.g., they may not afford the use of a combination of sensors with different technologies in the same measurement bench or to conceive different spatial geometries for the placing of the sensors, themselves, reducing the overall flexibility.

(2) Acquisition cards have prefixed characteristics that could not match the exigencies of new configurations. Hardware upgrades are usually impossible.

(3) The PC-based architectures are required to be installed in lab facilities. This requires moving the animals away from their natural environment. This prevents the possibility of in-field activity and could even introduce a bias.

(4) The software for the data elaboration available on the market need an operative system as Windows or MacOS to run. This increases the overall cost of the system.

(5) The overall dimensions are usually big.

(6) The overall costs of these systems could be extremely high.

A custom system based on the Raspberry-Pi allows overcoming these drawbacks. In fact:

(1) It allows designing of specific configurations (combination of sensors with different technologies and different spatial geometries for the placing of the sensors),

(2) It avoids buying a specific acquisition card.

(3) It allows the use of batteries to supply the system allowing in field acquisition campaigns.

(4) Using a release of Linux, it avoids use of proprietary OSs.

(5) It allows for direct programming by the user; therefore, it is possible to develop both the standard algorithms already used for this kind of research and new statistical functions that could show new interesting parameters for the study of these animals.

(6) The overall dimensions are extremely reduced with respect to a PC-based system.

(7) The costs are very low.

Table 1 shows the comparison between the costs of the Raspberry-based system with a PC one.

Table 1. Costs comparison between the Standard and the Raspberry system.

\begin{tabular}{cccc}
\hline Raspberry-Pi System & \multicolumn{2}{c}{ Standard System } \\
\hline Hardware & $\sim 75 €$ & Hardware & From 500 to $10,000 €$ \\
Software & Free & $\begin{array}{c}\text { Software (Operative System) } \\
\text { OS + elaboration tool) }\end{array}$ & From 500 to $3,500 €$ \\
Total & $\sim 75 €$ & Total & From 1,000 to $13,500 €$ \\
\hline
\end{tabular}

Despite the clear disadvantage to develop the software in house, the costs of a Raspberry-Pi system are extremely lower, about $1 \%$, than commercial ones.

The most important drawback of a custom system, especially if used in harsh environment, could be the reliability.

To verify if the system was able to work at low temperatures, some preliminary tests were performed in laboratory using a climate chamber. The temperature was decreased to $-20^{\circ} \mathrm{C}$ (that is at least 10 degrees under the lower operative temperature during the measurements season). In order to verify the correct activity of the board, a stress test script, designed to push the CPU at 100\% of its duty cycle, has been loaded on the Raspberry Pi and executed for all the duration of the test. The next graphs show three examples of the test done.

Figure 10 shows the temperature of the chamber, of the CPU and of the board. The first has been measured by the embedded sensor, the temperature of the CPU has been obtained by the internal sensor and the temperature of the board has been obtained by a PT100 sensor directly mounted on the board. 


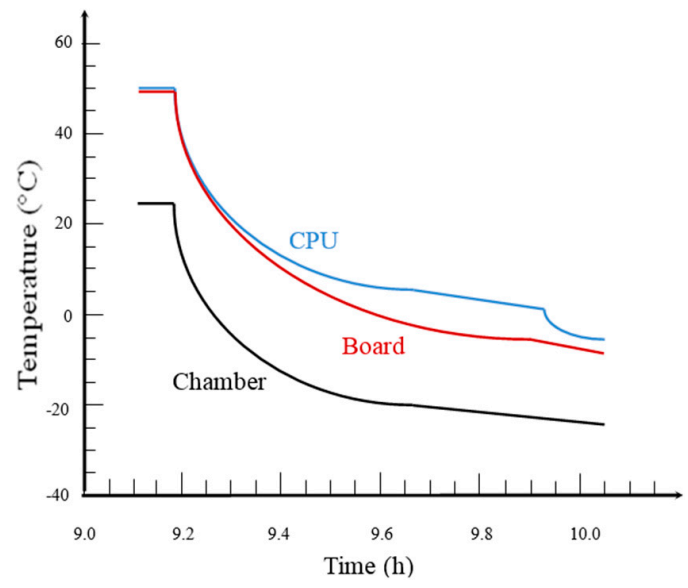

Figure 10. Temperature of climate chamber, CPU and board.

The figure shows that, jointly with the temperature of the chamber, the temperature of the CPU and of the board constantly decrease, but the device activity is always ensured.

Figure 11 presents an image of the board taken with an infrared camera that shows the uniformity of the temperature on the board [31].

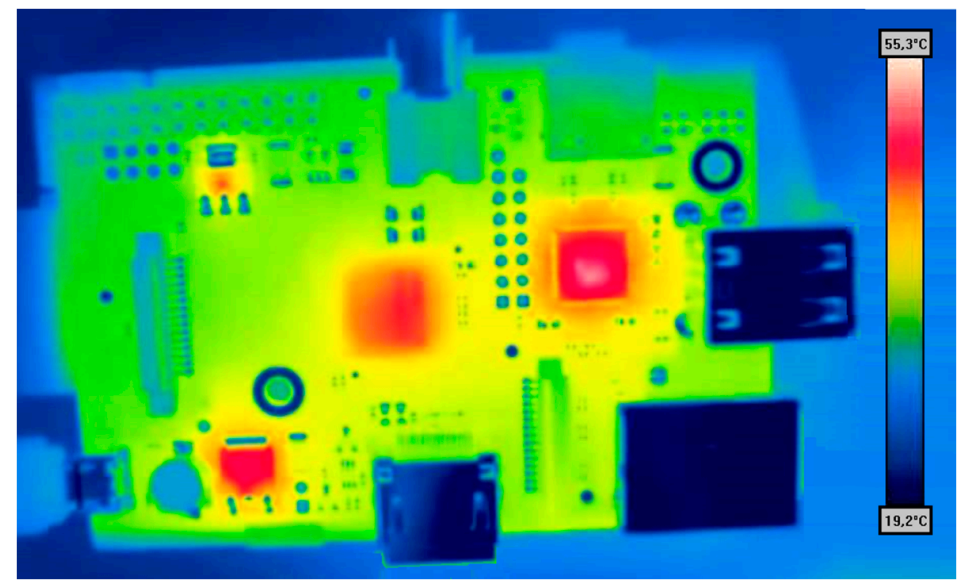

Figure 11. Temperature on the board caught using an infrared camera.

The second test shows the current consumption of the Raspberry as a function of the time (Figure 12).

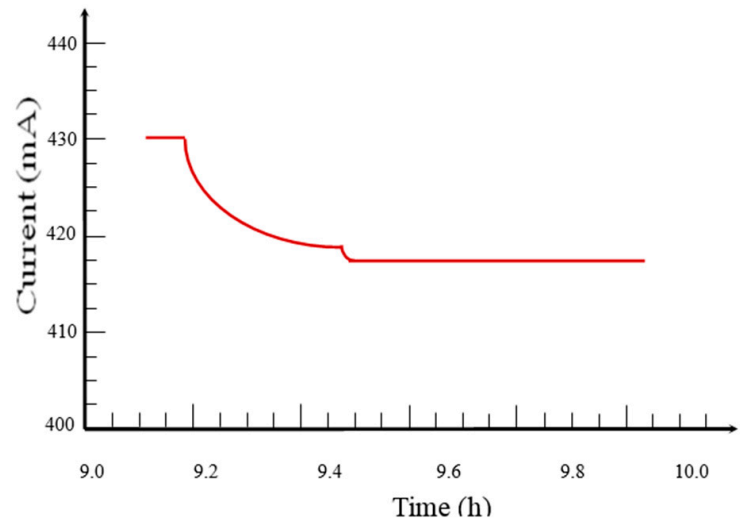

Figure 12. Current consumption as a function of the variation of time. 
After a brief transitory, the current consumption reaches a stable value that ensures the regular activity of the Raspberry.

Figure 13 shows the current consumption of the Raspberry as a function of the CPU temperature.

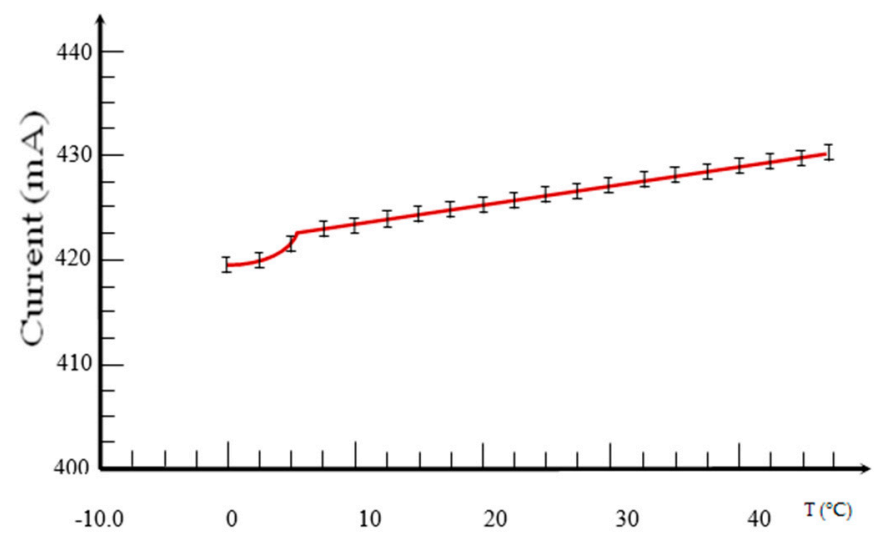

Figure 13. Current consumption as a function of the variation of the CPU temperature.

The plot clearly shows that the power consumption, through the current flow, rise accordingly with the board's temperature. These tests have been made to confirm that the Raspberry Pi is able to behave correctly even in extreme operating conditions. For the sake of consistency, we repeated the test on five boards, which, tested in the same configuration, with the same stress test script, shown concordant results, always ensuring the full efficiency of the board.

Testing the device in the field, requires a battery pack capable of providing the right power for the system. The power consumption of our setup has been experimentally measured, and it is about $4 \mathrm{~W}$; for a comparison, the power consumption of other commercial systems is around $130 \mathrm{~W}$. We tested, with a $12 \mathrm{~V}, 24 \mathrm{Ah}$ battery, that we were able to operate our setup for more than a week. We planned a campaign duration of seven days $(168 \mathrm{~h})$ with a single battery. The test was run in a climate chamber. For comparison, the same power supply, modified to power a classic PC-based setup with an AC/DC converter, would ensure an autonomy of just $10 \mathrm{~h}$.

After this preliminary lab phase, the device has been tested in the field confirming the Raspberry Pi reliability.

\section{Experiment, Results and Discussions}

Marine and freshwater Arctic invertebrates relevant to ecological studies were chosen for the experiments [32]. We analyzed the activity of the Gammarus Setosus that is a marine invertebrate (order of the amphipods). This animal commonly lives in intertidal zones and is a useful biomarker for pollution [33,34]. In this study, we applied a typical chronobiological design [35,36].

\subsection{Animals}

Gammarus setosus ( $n=20$ ) were collected in June and July 2015 (CNR and NP Summer Campaign 2015 Pasquali V. and Granberg M.) during the low tide from the coastal area on the Kongsfjorden, Spitsbergen Svalbard $\left(78^{\circ} 55^{\prime} 40^{\prime \prime} \mathrm{N}, 11^{\circ} 54^{\prime} 22^{\prime \prime} \mathrm{E}\right)$, using a hand-nest and stored in a $10 \mathrm{~L}$ plastic tank. Permits to conduct this research were issued by the Governor of Svalbard (Fieldwork 2015, RIS-ID 10211, Granberg M.).

\subsection{Experimental Procedure}

After capture, animals were carried to the laboratory where they were randomly selected and individually transferred into a $1 \mathrm{~L}$ plastic flask at a temperature of $10 \pm 1{ }^{\circ} \mathrm{C}$ that was exposed to continuous light (LL). The aquaria were not provided with pumps for circulation and filtration 
of the water because of possible interference with the electronic monitoring system. Water was changed every three days to maintain normal levels of oxygen, salinity, and $\mathrm{pH}$. The seawater used for replenishment was obtained from KingsBay Marine Laboratory (Ny-Alesund, Svalbard). No food was provided during the tests in order to prevent synchronization of activity rhythms with feeding time, as previously reported in marine decapods and fish held in similar laboratory conditions [37]. Animals were monitored continuously for 15 days; Figure 14 shows the complete set-up.

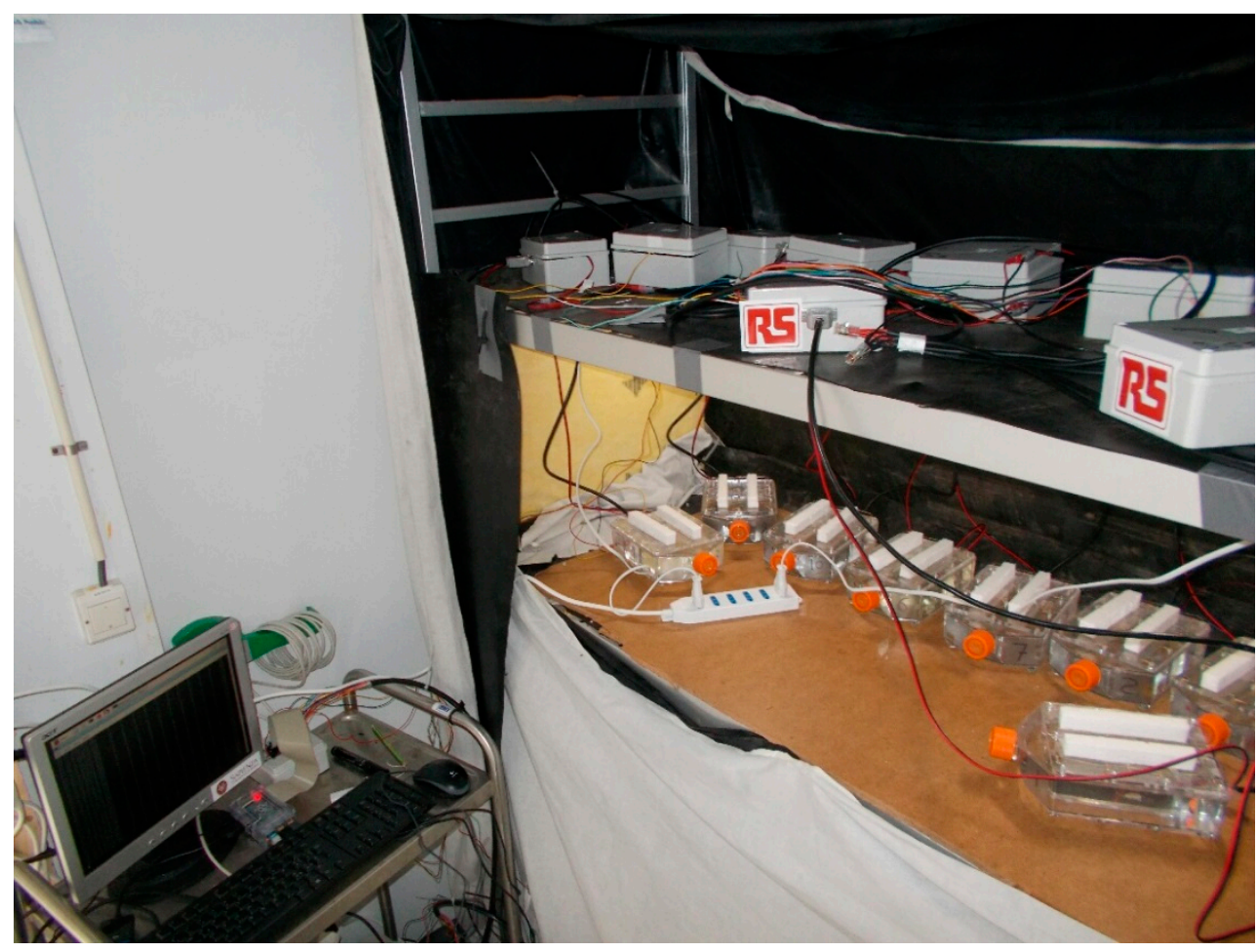

Figure 14. The complete set-up inside the cold room.

\subsection{Results and Discussion}

Chronobiologic parameters were calculated with Lomb-Scargle periodograms to obtain the length of the circadian period (between 20 and $28 \mathrm{~h}$ ) and the tidal period (between 10 and $14 \mathrm{~h}$ ). Power spectra analysis by FFT was used to observe the peaks and their magnitudes (corresponding to amplitude) at circadian and tidal periods. For the circadian periodicity under LL, only four out of 15 animals showed circadian rhythms, and these had a period length of $23.2 \pm 2.8 \mathrm{~h}$ (Mean $\pm \mathrm{SD}$ ). Tidal periodicity was also calculated and analysed; and we observed a period of $12.0 \pm 1.1 \mathrm{~h}$ (Mean $\pm \mathrm{SD}$ ) for only nine animals that showed tidal rhythms. Spectral analysis confirmed the presence of the circadian periods lower than twenty-four hours, and the presence of tidal rhythms. The amplitude of the circadian rhythm is $6.7 \% \pm 4.0 \% \mathrm{rPSD}$ (Mean $\pm \mathrm{SD}$ ), and the amplitude of the tidal rhythm is $6.9 \% \pm 4.3 \% \mathrm{rPSD}$ (Mean $\pm \mathrm{SD})$.

From a chronobiological point of view, the results show that this device can be useful for recording and characterizing chronobiologic parameters of these and other animals, not only in the circadian range, but also the ultradian (see reference [38] where different methodological approaches to study these rhythms is shown). This species has never been studied before in this way, and it could be an interesting model for studying the biological rhythms, particularly in marine high arctic animals.

From an electronic point of view, many challenges have been faced and overcome, and we have compiled a list of obtained goals:

(1) The reliability offered by the Raspberry-Pi is certified by field tests carried out for many days and has demonstrated that the Raspberry can be used to fabricate an instrument suitable for extremely 
harsh environments, such as those in Arctic and Antarctic. Consequently, this technology presents new opportunities for researchers working in similarly difficult situations, e.g., in outer space.

(2) The challenge to reduce the dimensions and the weight with respect to a PC-based, has been accomplished. This is also extremely important for various research where the weight and the dimensions are a variable fundamental for keeping shipment costs low.

(3) The device, which works @ 12 Vdc; therefore, it can be supplied by a battery as in our case. This allows one to place the device directly in the field without the mains, as contrasted with a PC-based system. This allow researchers to leave the animal in its natural environment without disturbing it.

(4) The use of the Raspberry allows researchers to conceive of new hardware for specific tasks.

(5) The possibility to directly program the device increases the overall flexibility of the instrument. In fact, new algorithms can be conceived by researchers to find new information. This feature is not always present in commercial tools that provide only predetermined functions.

\section{Conclusions}

The article shows the development and application of a new device based on the Raspberry-Pi to monitor the locomotor activity of small marine invertebrates. The device has been conceived to emulate more costly and complex commercially available systems. The use of IR sensors for the continuous monitoring of the activity of the animal and the use of specific software for data analysis is similar to that done in commercial alternatives but is performed at a lower cost. Apart from cost, the great advantage of our system is that the use of the Raspberry-Pi allows increasing overall flexibility, allowing creation of specific hardware configurations or specific analysis routines not provided by commercial systems. Moreover, the substitution of the computer with the Raspberry allows the reduction of the overall dimensions compared to a classical system, and thus reduces the shipping costs, which is an important factor for in-field research. This project demonstrates how the overall reduction of costs and the increase in performance flexibility may increase the interest towards this kind of research.

Acknowledgments: This work was supported by Svalbard Science Forum (Arctic Field Grant 2015), Consiglio Nazionale delle Ricerche, Confucio Institute-SAPIENZA Università di Roma, $6^{\circ}$ Comunità Montana del Velino, G.S.A. srl in the person of Giancarlo Colangeli, OPTEK Inc., Carrollton, TX, USA, Millennium Datawere srl Rivalta Scrivia-Italia and Silco srl Torino. The help and cooperation of the officers and persons of the CNR D.T.A. Department and Italian Arctic Base "Dirigibile Italia" (in the person of E. Brugnoli, V. Vitale, A. Viola, E. Liberatori) are gratefully acknowledged. Thanks also to the staff of Kings Bay Marine Laboratory, City, Country and Steve Coulson for his useful advice. A special thanks to Robert Gramillano, undergraduate researcher at University of Illinois at Urbana-Champaign (UIUC), for the precious help in writing.

Author Contributions: V. Pasquali, F. Leccese and Cagnetti conceived and designed the experiments; V. Pasquali and M. Granberg performed the experiments; R. Gualtieri and G. D'Alessandro analyzed the data, D. Hazlerigg, and F. Leccese and M. Cagnetti contributed reagents/materials/analysis tools. All authors wrote the paper.

Conflicts of Interest: The authors declare no conflict of interest.

\section{References}

1. Mancinelli, G.; Pasquali, V. Body size-related constraints on the movement behaviour of the arctic notostracan Lepidurus arcticus (Pallas, 1973) under laboratory conditions. Rend. Lincei 2016, 27, 207-215. [CrossRef]

2. Naylor, E. Chronobiology of Marine Organisms; Cambridge University Press: Cambridge, UK, 2010.

3. Elliott, E.; Manashirov, S.; Zwang, R.; Gil, S.; Tsoory, M.; Shemesh, Y.; Chen, A. Dnmt3a in the medial prefrontal cortex regulates anxiety-like behavior in adult mice. J. Neurosci. 2016, 36, 730-740. [CrossRef] [PubMed]

4. De Bruin, N.M.; Schmitz, K.; Schiffmann, S.; Tafferner, N.; Schmidt, M.; Jordan, H.; Parnham, M.J. Multiple rodent models and behavioral measures reveal unexpected responses to FTY720 and DMF in experimental autoimmune encephalomyelitis. Behav. Brain Res. 2016, 300, 160-174. [CrossRef] [PubMed] 
5. Aarts, E.; Maroteaux, G.; Loos, M.; Koopmans, B.; Kovaçevic, J.; Smit, A.B.; Verhage, M.; van der Sluit, S. The light spot test: Measuring anxiety in mice in an automated home-cage environment. Behav. Brain Res. 2015, 294, 123-130. [CrossRef] [PubMed]

6. Bernadou, A.; Ruther, J.; Heinze, J. Avoid mistakes when choosing a new home: Nest choice and adoption of leptothorax ant queens. J. Insect Physiol. 2015, 79, 88-95. [CrossRef] [PubMed]

7. Ghezzi, D.; Arzuffi, P.; Zordan, M.; Da Re, C.; Lamperti, C.; Benna, C.; Uziel, G. Mutations in TTC19 cause mitochondrial complex III deficiency and neurological impairment in humans and flies. Nat. Genet. 2011, 43, 259-263. [CrossRef] [PubMed]

8. Noguchi, T.; Lo, K.; Diemer, T.; Welsh, D.K. Lithium effects on circadian rhythms in fibroblasts and suprachiasmatic nucleus slices from Cry knockout mice. Neurosci. Lett. 2016, 619, 49-53. [CrossRef] [PubMed]

9. Basu, P.; Wensel, A.L.; McKibbon, R.; Lefebvre, N.; Antle, M.C. Activation of M1/4 receptors phase advances the hamster circadian clock during the day. Neurosci. Lett. 2016, 621, 22-27. [CrossRef] [PubMed]

10. Aguzzi, J.; Sbragaglia, V.; Sarriá, D.; García, J.A.; Costa, C.; Río, J.D.; Sardà, F. A new laboratory radio frequency identification (RFID) system for behavioural tracking of marine organisms. Sensors 2011, 11, 9532-9548. [CrossRef] [PubMed]

11. Last, K.S.; Hobbs, L.; Berge, J.; Brierley, A.S.; Cottier, F. Moonlight Drives Ocean-Scale Mass Vertical Migration of Zooplankton during the Arctic Winter. Curr. Biol. 2016, 26, 244-251. [CrossRef] [PubMed]

12. Proietti, A.; Panella, M.; Leccese, F.; Svezia, E. Dust Detection and Analysis in Museum Environment based on Computational Intelligence. Measurement 2015, 66, 62-72. [CrossRef]

13. Zordan, M.A.; Benna, C.; Mazzotta, G. Monitoring and analyzing Drosophila circadian locomotor activity. Methods Mol. Biol. 2007, 362, 67-81. [PubMed]

14. Cagnetti, M.; Leccese, F.; Trinca, D. A New Remote and Automated Control System for the Vineyard Hail Protection Based on ZigBee Sensors, Raspberry-Pi Electronic Card and WiMAX. J. Agric. Sci. Technol. B 2013, 3, 853-864.

15. Leccese, F.; Cagnetti, M.; Trinca, D. A Smart City Application: A Fully Controlled Street Lighting System Isle Based on Raspberry-Pi Card, ZigBee Sensor Network and WiMAX. Sensors 2014, 14, 24408-24424. [CrossRef] [PubMed]

16. D'alessandro, G.; de Bernardis, P.; Masi, S.; and Schillaci, A. Common-mode rejection in Martin-Puplett spectrometers for astronomical observations at millimeter wavelengths. Appl. Opt. 2015, 54, 9269-9276. [CrossRef] [PubMed]

17. Gualtieri, R.; Battistelli, E.S.; Cruciani, A.; de Bernardis, P.; Biasotti, M.; Corsini, D.; Gatti, F.; Lamagna, L.; Masi, S. Multi-mode TES Bolometer Optimization for the LSPE-SWIPE Instrument. J. Low Temp. Phys. 2016, 184, 527-533. [CrossRef]

18. OPTEK, OP298B Datasheet. Available online: http://optekinc.com/partNumberSearch.aspx?partNumber= op298 (accessed on 22 July 2016).

19. OPTEK, OP598B Datasheet. Available online: http://optekinc.com/partNumberSearch.aspx?partNumber= op598 (accessed on 22 July 2016).

20. Texas Instruments, MC3303 Datasheet. Available online: http://www.ti.com/product/mc3303 (accessed on 21 March 2015).

21. Texas Instruments, 74LS21 Datasheet. Available online: http://www.futurlec.com/74LS/74LS21.shtml (accessed on 21 March 2015).

22. Texas Instruments, LM555 Datasheet. Available online: http://www.ti.com/lit/ds/symlink/lm555.pdf (accessed on 21 March 2015).

23. Pasquali, V.; D'Alessandro, G.; Gualtieri, R.; Leccese, F. A new Data Logger based on Raspberry-Pi for Arctic Notostraca Locomotion Investigations. Measurements 2016, submitted.

24. ISO/IEC 9899:TC2 WG14/N1124 Committee Draft—6 May 2005. Available online: http://www.open-std. org/jtc1/sc22/wg14/www/docs/n1124.pdf (accessed on 8 September 2016).

25. IFFL LINUX Freedom. Available online: http://www.lffl.org/2014/09/xming-avviare-applicazioni-linuxremoto-windows.html (accessed on 8 September 2016).

26. Pasquali, V. Raw Data Used for the Article. Available online: http://dx.doi.org/10.5281/zenodo.61747 (accessed on 13 September 2016). 
27. Low-Zeddies, S.S.; Takahashi, J.S. Chimera analysis of the clock mutation in mice shows that complex cellular integration determines circadian behavior. Cell 2001, 105, 25-42. [CrossRef]

28. Lomb, N.R. Least-squares frequency analysis of unequally spaced data. Astrophys. Space Sci. 1976, 39, 447-462. [CrossRef]

29. Scargle, D. Studies in astronomical time series analysis. II-Statistical aspects of spectral analysis of unevenly spaced data. Astrophys. J. 1982, 263, 835-853. [CrossRef]

30. Townsend, R.H.D. Fast calculation of the Lomb-Scargle periodogram using graphics processing units. Astrophys. J. Suppl. Ser. 2010, 191, 247-253. [CrossRef]

31. Caciotta, M.; Leccese, F.; Schirripa Spagnolo, G.; Cozzella, L. Automatic Industrial Electrical Circuit Firing Prevention using Infrared Termography. In Proceedings of the 20th IMEKO TC-4 International Symposium Measurement of Electrical Quantities, Benevento, Italy, 15-17 September 2014.

32. Calizza, E.; Costantini, M.L.; Rossi, D.; Pasquali, V.; Careddu, G.; Rossi, L. Stable isotopes and digital elevation models to study nutrient inputs in high-Arctic lakes. Rend. Lincei 2016, 27, 191-199. [CrossRef]

33. Magnusson, K.; Magnusson, M.; Östberg, P.; Granberg, M.; Tiselius, P. Bioaccumulation of 14 C-PCB 101 and 14 C-PBDE 99 in the marine planktonic copepod Calanus finmarchicus under different food regimes. Mar. Environ. Res. 2007, 63, 67-81. [CrossRef] [PubMed]

34. Ugolini, A.; Pasquali, V.; Baroni, D.; Ungherese, G. Behavioural responses of the supralittoral amphipod Talitrus saltator (Montagu) to trace metals contamination. Ecotoxicology 2012, 21, 139-147. [CrossRef] [PubMed]

35. Pasquali, V. Locomotor activity rhythms in high arctic freshwater crustacean: Lepidurus arcticus (Branchiopoda; Notostraca). Biol. Rhythm Res. 2015, 46, 453-458. [CrossRef]

36. Lincoln, G.A.; Johnston, J.D.; Andersson, H.; Wagner, G.; Hazlerigg, D.G. Photorefractoriness in mammals: Dissociating a seasonal timer from the circadian-based photoperiod response. Endocrinology 2005, 146, 3782-3790. [CrossRef] [PubMed]

37. Costa, C.; Aguzzi, J.; Chiesa, J.J.; Magnifico, G.; Cascione, D.; Rimatori, V.; Caprioli, R. Evidences on the transient disruption of Sabella spallanzanii (Polychaeta, Sabellidae) fan activity rhythm in laboratory constant darkness. Ital. J. Zool. 2008, 75, 337-344. [CrossRef]

38. Pasquali, V.; Anna, C.; Paolo, R. Circadian and ultradian rhythms in locomotory activity of inbred strains of mice. Biol. Rhythm Res. 2010, 41, 63-74. [CrossRef]

(C) 2016 by the authors; licensee MDPI, Basel, Switzerland. This article is an open access article distributed under the terms and conditions of the Creative Commons Attribution (CC-BY) license (http://creativecommons.org/licenses/by/4.0/). 